\title{
Transtornos Alimentares, Autoestima e a Técnica de Rorschach
}

\author{
Daniela Sopezki* \\ Cícero E. Vaz \\ Pontifícia Universidade Católica do Rio Grande do Sul, Porto Alegre, Brasil
}

\begin{abstract}
RESUMO
A pesquisa teve como objetivo avaliar a autoestima de mulheres com transtornos alimentares. A amostra se constituiu de 51 participantes distribuídas em três grupos: anorexia nervosa (G1), bulimia nervosa (G2) e controle (G3). Os instrumentos utilizados foram: a Técnica de Rorschach e Escala de Rosenberg, para avaliar a autoestima e um questionário para levantamento de dados sociodemográficos e características da amostra. O EAT-26 e o MINI foram utilizados para triar o G3. A análise estatística foi por meio do Teste ANOVA e do Teste Qui-quadrado, com índice de significância de $\leq 0,05$. Observou-se que os G1 e G2 apresentaram indicativos de baixa autoestima, autoimagem e imagem corporal negativas, prejudicadas por distorções no pensamento, comparados com o G3.
\end{abstract}

Palavras-chave: transtornos alimentares; autoestima; Rorschach.

\section{ABSTRACT \\ Eating Disorders, Self Esteem and Rorschach Technique}

The research had as an aim to evaluate the self-esteem in women with eating disorders. The sample was composed by 51 participants distributed in three groups: anorexia nervosa (G1), bulimia nervosa (G2) and control (G3). The instruments which were used were: the Rorschach technique and the Rosenberg Scale to evaluate self-esteem, and a questionnaire to survey the demographic and social data and the sample characteristics. The EAT-26 and the MINI were used to select G3. The statistics analysis was made through ANOVA and the Chi-Square tests, with an accepted rate of significance of 0,05 . G1 and G2 presented indicators of low self-esteem, negative self-image and body image, harmed by distortions in the thought, compared to G3.

Keywords: eating disorders; self-esteem; Rorschach.

Os transtornos alimentares envolvem os quadros de anorexia nervosa (AN), bulimia nervosa (BN), o transtorno da compulsão alimentar periódica (TCAP) e ainda os transtornos alimentares sem outra especificação (TASOE) (DSMIV-TR, 2002). Essa área de estudo tem se especializado muito e constatado a multifatoriedade presente nesses quadros, o que demanda um atendimento global das pacientes.

Nos últimos meses esse tema tem sido encarado de forma mais séria, tendo em vista alguns episódios muito graves envolvendo modelos e adolescentes acometidas por esses transtornos que acabaram levadas à morte.
Poucos transtornos psiquiátricos se distribuem de forma tão assimétrica - em relação ao gênero - quanto os transtornos alimentares, com apenas $10 \%$ dos casos ocorrendo no sexo masculino (Melin \& Araújo, 2002), com uma prevalência média de relação homemmulher de 1:10 e até de 1:20 (Klein \& Walsh, 2004).

Esses transtornos possuem uma etiologia multifatorial, composta de predisposições genéticas, socioculturais e vulnerabilidades biológicas e psicológicas. Entre os fatores predisponentes estão os traços de personalidade (Morgan, Vecchiatti, \& Negrão, 2002), em especial, a baixa autoestima (McGee, 2000; Ghaderi \& Scott, 2001). 
Em ambos os casos, a baixa autoestima e a insatisfação com a imagem corporal são fatores de risco para transtornos alimentares, levando a um julgamento de si indevidamente, baseada na forma física, cuja avaliação se encontra comprometida por uma distorção cognitiva da percepção da autoimagem, debilitada por práticas inadequadas de controle de peso e comportamentos alimentares anormais (Fairburn, Doll, Davies, O’Connor, 1997; Fairburn, Zafra, Boneca, Welch, 1999).

Todavia, segundo Morgan, Vecchiatti e Negrão (2002) é necessária uma interação entre os fatores de risco e outros eventos precipitantes para a configuração do quadro de anorexia ou bulimia. E ainda o curso transitório ou crônico de um transtorno alimentar está relacionado à persistência de distorções cognitivas, à ocorrência de eventos vitais significativos e a alterações secundárias ao estado de desnutrição.

\section{AUTOESTIMA E TRANSTORNOS ALIMENTARES}

Para Rosenberg (1989), a autoestima expressa um sentimento ou uma atitude de aprovação ou de repulsa de si mesmo e até que ponto o sujeito se considera capaz, significativo, bem sucedido e valioso. É o juízo pessoal de valor expresso nas atitudes que o indivíduo tem consigo mesmo.

Há muita controvérsia na definição da autoestima. Mruck (1998) ressalta que existem quatro formas distintas de defini-la, dependendo do enfoque dado pelo autor e sua linha teórica.

A autoestima é um dos componentes do autoconceito e manifesta-se pela aceitação de si mesmo como pessoa e por sentimentos de valor pessoal e de autoconfiança. Ela constitui um dos determinantes mais importantes do bem-estar psicológico e do funcionamento social (Salmivalli, Kaukiainen, Kaistaniemi, \& Lagerspetz, 1999) e tem sido considerado como importante indicador da saúde mental (Andrade \& Angerami, 2001). André e Lelord (2000) descrevem que a autoestima assenta-se em três grandes pilares: a autoconfiança, a imagem de si próprio e o gostar de si mesmo, sendo este último o primeiro pilar e o elemento mais importante.

A autoestima é um fator predisponente, precipitador e perpetuador do desenvolvimento e manutenção dos transtornos alimentares, segundo Newn, Bell e
Thomas (2003). Existem diversos estudos sugerindo que a baixa autoestima ou autoavaliação negativa são fatores de risco importantes para o desenvolvimento tanto da AN quanto da BN (McGee, 2000; Ghaderi \& Scott, 2001; Morgan et al., 2002).

É amplamente aceito que os fatores de risco para a bulimia nervosa, principalmente a insatisfação com o corpo são dependentes de fatores culturais. Jaeger, Ruggiero, Edlund, Gómez-Perreta, Lange et al. (2002) avaliaram a insatisfação corporal e outros fatores de risco para a bulimia em doze diferentes países para revelar suas inter-relações. Os pesquisadores verificaram que quanto maior era a insatisfação corporal menor era a autoestima. Abreu e Cangelli (2004) identificaram que as mulheres com BN apresentam uma autoestima flutuante, ou seja, quando têm a sensação de controle, sua autoestima é alta, quando descontrolam-se, têm compulsões e purgações e sua autoestima se rebaixada.

Estudos com amostras não clínicas, de universitárias (Kugu, Akyuz, Dogan, Ersan, \& Izgic, 2006) também apontaram diferenças significativas entre os grupos. Nas garotas que apresentaram mais sintomas alimentares anormais (medidos pelo EAT-26) foi pontuada uma autoestima mais baixa. Os autores (Binford $\&$ Grange, 2005) compararam dois grupos: bulimia e transtorno alimentar sem outra especificação, com características não purgativas e identificaram que no primeiro grupo a autoestima era mais baixa, bem como tinham mais preocupações com o peso, a forma e a alimentação.

Apesar da extensa literatura disponível acerca do universo psicológico dos T.A., poucos estudos têm utilizado o Rorschach na investigação da personalidade dos pacientes acometidos por esses transtornos (Rossi, et al., 2000). Em um levantamento bibliográfico feito por Oliveira-Cardoso e Santos (2012), no qual utilizaram três bases de dados (Medline, Lilacs e Scielo), com o cruzamento das palavras-chave: bulimia nervosa, anorexia nervosa, transtornos alimentares e Rorschach, no período de 1997 a 2011. Foram encontrados apenas quinze artigos, dentre os quais cinco focalizavam o transtorno do comer compulsivo, logo é preciso contribui com esse campo.

Este estudo pretende aprofundar e contribuir com dados que se agreguem aos conhecimentos relativos 
às características da personalidade das mulheres acometidas pelos transtornos alimentares. $\mathrm{O}$ estudo teve como objetivo comparar a autoestima de mulheres com anorexia nervosa (G1), mulheres com bulimia nervosa (G2) e mulheres de um grupo controle (G3). $\mathrm{O}$ uso da técnica do Rorschach objetivou corroborar resultados de aspectos da personalidade, no caso a autoestima, e estimular sua aplicação quando o caso em questão envolve tamanha complexidade para o entendimento da personalidade, como é a situação dos transtornos alimentares e também contribuir para o campo científico com mais um estudo na área, que ainda está em desenvolvimento, embora a técnica seja utilizada na prática clínica.

\section{MÉTODO}

\section{Amostra}

A amostra foi selecionada por conveniência e se constituiu de 51 mulheres, distribuídas em três grupos $(\mathrm{G} 1=15, \mathrm{G} 2=17$ e $\mathrm{G} 3=19)$. A maioria $(\mathrm{n}=42)$ das voluntárias era proveniente da cidade de Porto Alegre e Grande Porto Alegre, algumas $(n=9)$, de cidades do interior do estado do Rio Grande do Sul, porém residentes em Porto Alegre.

Os grupos de transtornos alimentares foram compostos por mulheres que estavam em tratamento nas três instituições onde foi realizada pesquisa de campo. Das cinco instituições oficiais existentes em Porto Alegre, duas não se dispuseram a aceitar a execução da pesquisa em suas dependências.

Para a seleção da amostra de pacientes com AN e $\mathrm{BN}$ foram consultados os profissionais dessas instituições que tratavam tais casos para que encaminhassem as pacientes, que ainda mantinham tais diagnósticos, conforme suas avaliações e seus prontuários clínicos.

O grupo controle foi escolhido por conveniência, de duas instituições educacionais: uma de Ensino Médio (de classe média a baixa) e a outra universitária (de classe média a alta). Para a triagem das participantes desse grupo, quanto a não apresentarem comportamento alimentar anormal, foi aplicado o "Eating Attitudes Test” (EAT-26) e o MINI (Mini International Neuropsychiatric Interview).

O pareamento entre os grupos foi feito por faixa etária, escolaridade e renda. A média de idade foi de $21,6(\mathrm{DP}=6,4)$ e foi observada distribuição semelhante quanto ao estado civil, à escolaridade, à ocupação e à renda de todos os grupos.

\section{Instrumentos}

1) Técnica de Rorschach. É uma técnica projetiva elaborada por Hermann Rorschach em 1921, para avaliar, no sentido geral, a estrutura da personalidade e o funcionamento de seus psicodinamismos (Salmon, Arnold, \& Collyer, 1972; Vaz, 1997).

Consiste em 10 cartões contendo manchas de tinta. Esses estímulos não estruturados mobilizam processos de percepção, associação, projeção e de expressão verbal na medida em que o examinando verbaliza o que percebe. Sobre as verbalizações emitidas, transformadas em variáveis de dados quantitativos e qualitativos, elaboraram-se sistemas de escores que permitem estudos de fidedignidade e validade, além de estudos normativos disponíveis no Brasil (Nascimento, 2007; Vaz, 1997, 2006).

Segundo Gonçalves e Pereira (2001), considera-se como resposta quantitativa toda interpretação que satisfaça os parâmetros de localização, determinantes e conteúdos. Já as respostas qualitativas são exclamações, impressões, comentários intelectuais, simetrias, adornos, críticas ou digressões em torno da qualidade dos estímulos apresentados nas diferentes pranchas e/ou cartões.

Para avaliar a autoestima, foram analisadas as respostas atribuídas ao cartão $\mathrm{V}$, que representa o cartão da expressão e adaptação à realidade (Anzieu), e manifestações de ordem narcísica (Chabert). Segundo Vaz (2006) é o cartão que expressa o próprio self do examinando, a autoimagem.

Nesse estudo, as variáveis do Rorschach utilizadas para avaliar um bom ajustamento com a autoimagem e com o self, ou seja, a autoestima foram as seguintes: número de respostas de conteúdos humanos $(\mathrm{H})$ e detalhes humano (Hd) (quanto mais as respostas forem semelhantes aos seres humanos indica maior autoaceitação, aceitação de suas características, o que pode implicar na possibilidade de estabelecer bons relacionamentos, o oposto é verdadeiro). E número de respostas forma precisa $(\mathrm{F}+)$ que significa a percepção dos aspectos evidentes das experiências. O contrário ( $\mathrm{F}-$ ou $\mathrm{F}=/$-) indica distorção na percepção dos fatos e das situações, e ainda contato precário com a realida- 
de, reduzido interesse pelos estímulos externos, tratando-se de indivíduo intensamente voltado para aspectos internos.

$\mathrm{E}$ as respostas dadas ao cartão $\mathrm{V}$ envolvendo: referência à cor preta (que significa uma tendência mais depressiva do estado de humor, alguma angústia), crítica (dificuldade de relação com o outro) e choques aos cartões (dificuldade de elaboração, de formação de um sentido ou significado á experiência).

2) Escala de Rosenberg (EAR). Desenvolvida por Rosenberg (1989) um dos instrumentos mais utilizados para avaliação da autoestima (Heatherton \& Wyland, 2003). No Brasil, esse instrumento foi originalmente adaptado e validado para pesquisa por Hutz (2000) e essa versão tem sido utilizada por vários pesquisadores, inclusive nesse estudo. Avanci, Assis, Santos e Oliveira (2006) publicaram uma versão dessa escala adaptada para adolescentes.

Sbicigo, Bandeira e Dell'ágio (2010) investigaram as propriedades psicométricas da EAR para adolescentes, de nove cidades brasileiras. Os participantes responderam a uma versão da EAR adaptada para o Brasil, por Hutz (2000). As análises de consistência interna realizadas por meio do coeficiente alfa de Cronbach, confiabilidade composta e variância extraída indicaram bons valores de fidedignidade.

Hutz e Zanon (2011) atualizaram as propriedades psicométricas da EAR, adaptada por Hutz (2000), com uma amostra de 1151 estudantes da região sul do Brasil. A consistência interna da escala (alfa de Cronbach $=0,90$ ) foi satisfatória, em concordância com o resultado original de Rosenberg (1989).

A EAR é uma medida unidimensional constituída por dez afirmações relacionadas a um conjunto de sentimentos de autoestima e autoaceitação que avalia a autoestima global. Os itens são respondidos em uma escala tipo Likert de quatro pontos variando entre concordo totalmente, concordo, discordo e discordo totalmente. Para esse estudo foi calculado o Alfa de Cronbach, para esse estudo, que resultou em 0,96.

3) EAT-26 (Eating Attitude Test). É um questionário mais utilizado em avaliações de comportamento de risco e sintomas de T.A., devido a sua facilidade de aplicação, eficiência, economia e não necessidade de treinamento para administração (Mintz \& O’Halloran,
2000). No Brasil, apenas a versão reduzida do EAT, conhecida por EAT-26, foi traduzida e validada $(\mathrm{Nu}-$ nes et al., 1994; 2005). A investigação das propriedades psicométricas do EAT-26 foi realizada por Bighetti (2003). O EAT-26 avalia os comportamentos alimentares inadequados, consta de uma ferramenta de autopreenchimento contendo 26 itens, com respostas em escala do tipo Likert ( 0 = nunca; quase nunca; ou poucas vezes; 1 = às vezes; $2=$ muitas vezes; $3=$ sempre), sendo que a pergunta de número 25 apresenta escore invertido. No Brasil, os estudos que avaliaram comportamento de risco, com essa escala, também encontraram prevalências preocupantes (Ferreira \& Veiga, 2008). O único estudo nacional de base populacional é o de Nunes, Barros, Olinto, Camey e Mari (2003) que avaliou uma amostra representativa de mulheres de Porto Alegre, encontrando 16,5\% da população com sintomas de T.A., segundo o EAT-26. Avaliando 1.807 crianças e adolescentes de 7 a 19 anos, em Minas Gerais, Vilella, Lamounier, Dellaretti, Neto e Horta (2004) encontraram 13,3\% com sintomas de TA, segundo o EAT-26.

Considera-se pontuação igual ou maior que 20 pontos sugestiva de comportamento alimentar de risco para transtornos alimentares e menor de 20 pontos ausência de transtorno alimentar (Garner, Garfinkel, 1979; Nunes, Bagatini, Abuchaim, Kunz, Ramos, Silva et al., 1994). A versão de Nunes, Camey, Olinto e Mari (2006) para o EAT-26 teve consistência interna (Alpha de Cronbach) de 0,75; o trabalho de Bighetti, F., Santos, Santos e Ribeiro (2004) validando outra versão em português do EAT-26 com adolescentes do sexo feminino encontrou Alpha de Cronbach de 0,82. Para fins de comprovação da adequação do instrumento à presente amostra, calculou-se a consistência interna do EAT-26, obtendo-se valores de alfa de Cronbach de 0,89 . Nesse estudo, a escala foi utilizada para triar o grupo controle.

4) MINI. É uma entrevista diagnóstica padronizada breve, é destinada à utilização na prática clínica e na pesquisa em atenção primária e em psiquiatria, e pode ser utilizada por clínicos após um treinamento rápido (de 1 a 3 horas). Muito utilizada na seleção de pacientes, segundo critérios internacionais, em estudos clínicos e epidemiológicos. É uma entrevista diagnóstica breve padronizada (15-30 minutos), com- 
patível com os critérios do DSM-IV e da CID-10. É organizado por módulos diagnósticos independentes e explora critérios obrigatórios, permitido, assim excluir o diagnóstico em caso de respostas negativas. Os índices de confiabilidade do MINI foram globalmente satisfatórios, mostraram qualidades psicométricas similares às de outras entrevistas diagnósticas padronizadas mais complexas, permitindo uma redução de $50 \%$ ou mais no tempo da avaliação (Amorim, 2000).

Nesse estudo foi utilizada como instrumento de triagem das participantes do grupo controle.

5) Questionário de dados sociodemográficos. Foi elaborado pela autora com a intenção de caracterizar a amostra em termos sócio-demográficos, alguns dados dos quadros psicopatológicos e familiares. O alfa de Crobach resultou em 0,53 , tendo sua validade reforçada pelos demais instrumentos.

\section{Procedimentos éticos}

Após a aprovação do projeto pela Comissão Científica da Faculdade de Psicologia da PUCRS e pelo Comitê de Ética em Pesquisa da mesma instituição, a pesquisa foi realizada nas instituições combinadas.

Foram marcadas entrevistas individuais com todas as participantes e após uma explanação sobre o estudo e o aceite formal das participantes, com a assinatura do Termo de Consentimento Livre e Esclarecido, iniciou-se a aplicação dos instrumentos.

\section{Procedimentos de coleta}

Todas pacientes vinculadas às instituições, que apoiaram a realização do estudo, foram convidadas pessoalmente a participar, houve apenas duas recusas.

No caso do grupo controle, aquelas que pontuaram comportamento alimentar anormal (segundo o EAT26) ou alguma psicopatologia (de acordo com o MINI) foram excluídos e não participaram da etapa seguinte: aplicação do Rorschach, Escala de Autoestima de Rosenberg e questionário, comum a todas participantes integrantes do estudo. Para as entrevistas, salas adequadas foram previamente agendadas nas próprias instituições.

\section{Procedimentos de análise dos dados}

Nesse estudo foi adotado o Sistema de Classificação de Klopfer, adaptado e validado por Vaz (1997, 2006). A classificação (Localizações, Determinantes e Conteúdos) dos protocolos do Rorschach foi realizada às cegas e sucessivamente por três juízes treinados na técnica, participantes do grupo de pesquisa no qual a autora estava inserida. Todos os protocolos foram codificados, quantitativamente e qualitativamente. $\mathrm{O}$ estudo de concordância entre dois juízes foi conferido por meio do coeficiente Kappa. O Kappa é uma medida de concordância interobservador e mede o grau de concordância além do que seria esperado unicamente pelo acaso. Quando houve discordância, os juízes por meio de uma discussão entre ambos se decidiam pela melhor codificação. Nos casos em que não houve um consenso entre os dois juízes, o terceiro juiz, expert na área, julgava a melhor codificação.

Foram utilizados para estudo estatístico a análise de variância (ANOVA), o teste t e o coeficiente de correlação de Pearson; e para todas as análises estatísticas, o nível de significância para aceitação das hipóteses alternativas $\leq 0,05$.

\section{RESULTADOS}

Em relação aos escores de autoestima, verificados pelo teste ANOVA, da Escala de Autoestima de Rosenberg, os dois grupos de transtornos alimentares apresentam médias e desvios padrões distintos do grupo controle, respectivamente $(\mathrm{G} 1=23,8$ e 7,2; $\mathrm{G} 2=23,4$ e 7,5 e $\mathrm{G} 3=33$ e 4,49) sendo assim, houve diferença significativa entre os grupos $(p=0)$, o que demonstra uma baixa autoestima nas mulheres acometidas pelos transtornos alimentares.

Esses dados conferem com o levantamento das variáveis verificadas pelo uso da Técnica de Rorschach tanto pela análise quantitativa das variáveis em estudo quanto às variáveis qualitativas (exemplo na tabela abaixo). 
Tabela 1

Fragmentos de verbalizações emitidas no Cartão $V$ do Rorschach aplicado em mulheres com bulimia nervosa, mulheres com anorexia nervosa e grupo controle

\begin{tabular}{|c|c|c|}
\hline Anorexia & Bulimia & Controle \\
\hline dois bichos mortos, é mais a carcaça. & $\begin{array}{l}\text { morcego, não gosto da cara dele, tem cara } \\
\text { de mal. }\end{array}$ & $\begin{array}{l}\text { um pássaro grande, tô vendo de cima, } \\
\text { voando. }\end{array}$ \\
\hline $\begin{array}{l}\text { uma borboleta, é preta diferente das } \\
\text { outras. }\end{array}$ & um incêndio, fogo, uma fogueira. & duas pessoas viradas de costas, deitadas. \\
\hline um bichinho estranho, feio... & uma borboleta, meio feia, meio gasta, velha. & $\begin{array}{l}\text { braços abertos, como se fosse uma pose } \\
\text { sacra, santa }\end{array}$ \\
\hline $\begin{array}{l}\text { morcego, parecido comigo, dorme de } \\
\text { dia e fica acordado de noite, preto. }\end{array}$ & $\begin{array}{l}\text { ai meu Deus! uma borboleta, é preta, toda } \\
\text { ela é preta, o que é difícil. }\end{array}$ & $\begin{array}{l}\text { um morcego por causa das patinhas, das } \\
\text { asas e porque ele tá voando. }\end{array}$ \\
\hline $\begin{array}{l}\text { um morcego, é parecido, as asas, } \\
\text { acho que é preto, feio. }\end{array}$ & $\begin{array}{l}\text { pés, de pessoas e bichos, corpos soterrados, } \\
\text { com os pés para fora. }\end{array}$ & $\begin{array}{l}\text { duas pessoas deitadas, uma de costas } \\
\text { para a outra. }\end{array}$ \\
\hline $\begin{array}{l}\text { Coxa assada, como eu sou comilona, } \\
\text { eu só enxergo comida. }\end{array}$ & $\begin{array}{l}\text { eu quando pratico bulimia, com raiva de mim, } \\
\text { olho no espelho ou deito na parede. }\end{array}$ & $\begin{array}{l}\text { borboleta, as asas grandes, batendo; e } \\
\text { uma coruja, pelo contorno do corpo. }\end{array}$ \\
\hline
\end{tabular}

Observou-se diferença também no número de respostas de conteúdos humanos $(\mathrm{H})$ e detalhes humanos (Hd) ( $\mathrm{p}=0,04$ e $\mathrm{p}=0,04$ respectivamente) indicando que no G1 e G2 e há menos projeções de respostas envolvendo conteúdos humanos, o que denota dificuldades de autoaceitação, aceitação de suas características, o que pode implicar na impossibilidade de estabelecer bons relacionamentos, porque os dados sugerem dificuldade de se identificar e em entrar em contato com o outro e manter relacionamentos mais profundos.

Tal dado se liga ao resultado das diferenças significativas encontradas entre as variáveis $\mathrm{F}+$ (forma precisa) e F- (forma de má qualidade) em ambos os grupos de mulheres com transtorno alimentar diferenciando-se do grupo controle, abaixo das porcentagens normais, que representa dificuldade da percepção dos fatos, dificuldade em captar os aspectos mais óbvios das situações, ou até distorção na análise dos fatos em função de prejuízo intenso na atenção e concentração, e ainda contato precário com a realidade, reduzido interesse pelos estímulos externos, tratando-se de indivíduo intensamente voltado para aspectos internos, baixa espontaneidade e inibição acentuada, caracterizando as reações como mais impessoais, demonstrando atitudes reprimidas e uma reduzida capacidade de precisão e controle do pensamento, com dificuldade de observação e concentração. Esses dados também são corroborados pelos escores do Rorschach que se referem às variáveis do tipo de vivência afetiva. Observou-se predomínio das vivências introversivas. Esses dados indicam uma preferência pelo isolamento ou relacionamento com menores grupos e uma prevalência do mundo imaginário sobre a realidade externa.

Estabelecendo-se um link entre os resultados da escala de autoestima com o significado simbólico do cartão V do Rorschach, é possível observar que o grupo controle verbaliza respostas mais estruturadas no cartão V (tabela II) e percebe-se um componente depressivo nas respostas das meninas com anorexia e bulimia, devido à referência à cor preta, choques ao cartão (“ai meu Deus") e críticas. E verbalizações que denotam uma autoimagem desqualificada, como: "mortos", "diferente", "estranho", "velha", "mal", entre outros, que sugerem a forma como a própria pessoa se percebe inconscientemente.

Ainda é possível relacionar essas variáveis com a seguinte questão do instrumento de dados sóciodemográficos: "está satisfeita com seu corpo atual?". Como esperado, ambos os grupos de transtornos alimentares estavam muito menos satisfeitas do que o grupo controle $(\mathrm{p}=0,00)$, sentem-se mais pressionadas por outras pessoas para ser magras $(\mathrm{p}=0,02)$ e possivelmente por isso também se pesam mais seguidamente $(p=0,00)$ e modificariam alguma parte do seu corpo $(\mathrm{p}=0,03)$.

O índice de massa corporal (IMC) do G1 estava um pouco abaixo da faixa normal e o dos demais grupos adequados $(p=0,00)$, a média de peso sonhado pelas primeiras era de $45 \mathrm{~kg}$, os demais de $51 \mathrm{~kg}$ ( $\mathrm{p}=$ $0,00)$, resultando num IMC ainda mais baixo $(\mathrm{p}=$ $0,01)$, no caso do G1. Por fim, as médias atribuídas para seus corpos foram 5,5 para G1, 4,6 para G3 e 7,8 
G3 $(\mathrm{p}=0,00)$, dados esses que ilustram e reforçam os critérios diagnósticos dessas psicopatologias.

\section{DISCUSSÃO}

Nesse estudo pôde-se constatar que há, nas mulheres com anorexia nervosa e bulimia nervosa há fortes indicativos de baixa autoestima, distorção no pensamento lógico, que perturba percepção da autoimagem e a imagem corporal, características da personalidade que reforçam suas insatisfações com o corpo e, consequentemente, abalam sua autoestima.

É possível que a tendência nas pacientes com transtornos alimentares a voltarem à atenção para o mundo interno dificulte o estabelecimento e manutenção de contatos sociais e ainda favorece essa relação obsessiva com o peso e a forma corporal. Como declaram Gorgati, Holcberg e Oliveira (2002), o senso de coesão do Self está comprometido nos pacientes com transtornos alimentares. Nessas pessoas, a identidade é fugaz e o ego revela-se frágil, resultado de experiências arcaicas do desenvolvimento, e como consequência, é provável que ataque negativamente a autoestima. Rosenberg (1989) relatou que a baixa autoestima implica autorrejeição, insatisfação consigo e desprezo por sua própria pessoa, percebido nos grupo dos transtornos alimentares. Possivelmente por isso também se pesam mais seguidamente, porque desejam menos peso e modificariam alguma parte do seu corpo, mesmo com o índice de massa corporal (IMC), no caso do G1, abaixo da faixa normal.

A diferença no número de respostas de conteúdos humanos e detalhes humanos, no Rorschach, com escores abaixo da média padrão normal, encontrados nos grupos de meninas com transtornos alimentares, denota a dificuldade de aceitação da autoimagem evidentes nesses quadros, corroboradas pelo estudo de Alba (2005), no qual identificou que, no caso das meninas com anorexia, havia maior incidência de respostas de conteúdos humanos descaracterizados, o que denota autoimagem fantasiada, baseadas em experiências fantasiadas e irreais, significativamente diferente de adolescentes considerados normais. Ainda em relação à AN, Garcia-Alba (2004) levantou, por intermédio do Rorschach, diferenças significativas nos sintomas depressivos e nas estratégias de enfrentamento dos pacientes com AN.
- O pensamento lógico, nas pacientes com TA estava prejudicado em seu aspecto de precisão e coerência sujeito a distorções cognitivas, inclusive na própria imagem corporal, sintoma presente nessas patologias, chamado de distorção do senso-percepção, dado que também foi encontrado por Smith et al. (1991) ao realizarem um estudo do tipo caso-controle com pacientes com BN, com o Método de Rorschach, no qual foi identificado nas pacientes com $\mathrm{BN}$ uma maior imprecisão perceptiva, pensamento desordenado, maior vulnerabilidade à invasão dos afetos, dificuldades nos relacionamentos interpessoais e autoimagem prejudicada, comparadas ao grupo controle. Sintomas semelhantes também foram encontrados por Smith, Hillard e Roll (1991), que ao delinear as características de personalidade em adolescentes com e sem $\mathrm{BN}$, por meio da aplicação do método de Rorschach, identificaram mais agressividade, depressão, comportamentos autopunitivos, pensamento mais desordenado, com imprecisões perceptivas e julgamento prejudicado no grupo com BN. Os autores observaram que os distúrbios cognitivos não se restringiam apenas aos setores da alimentação e do peso, o que sugere uma distorção mais ampla do funcionamento intelectual.

- Pacientes com BN foram também comparados com pacientes com depressão e com indivíduos sem diagnóstico clínico por Weisberg, Norman e Herzog (2006). Os autores, por meio da Técnica de Rorschach, mostraram que, diferentemente do grupo sem diagnóstico de depressão, humor disfórico, labilidade emocional, impulsividade, vivência de sobrecarga emocional, empobrecimento da precisão perceptiva e reduzido interesse por outras pessoas foram características presentes e semelhantes nos grupos das pacientes bulimicas e com depressão. A diferença encontrada entre esses dois últimos grupos foi: maior egocentrismo e narcisismo, presença mais acentuada de raiva e negatividade nas bulímicas, ao passo que na depressão observou-se maior introspecção e mais arbitrariedade nas percepções.

- Os grupos de transtornos alimentares estavam menos satisfeitas com sua autoimagem do que o 
grupo controle e se sentem mais pressionadas por outras pessoas para ser magras. Como identificaram Wallach e Lowenkopf (1986) há um conformismo exagerado ao pensamento coletivo, aos valores e padrões convencionais, podendo indicar necessidade de aprovação. E assim, como afirma Connan (2007) os relacionamentos interpessoais são temidos, porém, ao mesmo tempo, são marcados por um desejo intenso de aprovação social. Os resultados obtidos são consistentes com a literatura, que indica que mulheres com TA mostram características de ansiedade e padrões de evitação em seus relacionamentos com outros adultos (Ward, 2000).

O desenvolvimento da insatisfação com o próprio corpo poderia estar relacionado com a alta expectativa de serem rejeitadas pelo outro. Esse temor faria com que tais indivíduos dependessem exageradamente da aprovação social e da aceitação dos outros, tornando-os mais sensíveis às mensagens da sociedade relacionadas à aparência e aos valores positivos associados à magreza (Troise, 2006).

Em função dessa realidade, há uma preocupação em desenvolver programas preventivos de comportamentos alimentares anormais. O'Dea e Abraham (2000) criaram um programa de intervenção focando o aumento da autoestima. O modelo educacional tinha como objetivo ajudar uma amostra de adolescentes a ter mais satisfação com sua imagem corporal. Depois de doze meses os estudantes ainda mantinham autoestima, satisfação corporal e aceitação social adequados; aqueles que inicialmente pareciam estar sob risco de desenvolver algum transtorno alimentar também estavam adequados depois de um ano, comprovando a eficácia do projeto. No entanto, criar programas de prevenção para esses transtornos não é tarefa fácil tendo em vista que outros pesquisadores tentaram e não perceberam efeito (Ghaderi, Martensson, \& Schwan, 2005). Um programa de prevenção de transtornos alimentares deve comportar diversas variáveis tendo em vista sua etiologia multivariada, mas a autoestima certamente deve ser considerada, porque é fator de proteção para diversas psicopatologias.

\section{CONCLUSÃO}

Tendo em vista todos esses resultados, é evidente que o quadro de sofrimento psíquico das mulheres com anorexia nervosa e bulimia nervosa demanda um adequado acompanhamento psicoterapêutico. Nesse acompanhamento seria indicado incluir as pessoas com quem mais convivem essas pacientes, principalmente os familiares, para que seja proporcionado um diálogo mais claro sobre as necessidades dos membros acometidos pelo T.A., de modo a minimizar suas dificuldades nas relações interpessoais. Para essa demanda, é possível sugerir que diante da complexidade característica dos quadros de TA, os profissionais de saúde necessitam de conhecimentos específicos acerca do funcionamento psicodinâmico e de suas implicações na clínica, para que possam traçar estratégias mais eficazes de intervenção e como alternativa, um dos instrumentos mais consagrados que o psicólogo tem a sua disposição para compreender o funcionamento psíquico de um indivíduo, considerando as condutas intelectuais, os fatores de socialização, a relação com a realidade e sua dinâmica afetiva, é a Técnica de Rorschach.

Por fim, é ponderável salientar que esse estudo, assim como outros que envolvem pacientes com AN e $\mathrm{BN}$, tem algumas limitações. A primeira é a captação da amostra devido à baixa prevalência desses transtornos e à forte negação do problema. A segunda limitação foi a impossibilidade de se de poder separar em subgrupos diagnósticos, o que impede de se caracterizar e delimitar mais precisamente as sutis diferenças entre eles. No entanto, o estudo traz resultados bastante concisos, fundamentados pela própria literatura e a força dos instrumentos utilizados.

A produção bibliográfica relativa à organização e funcionamento da personalidade nos TA, codificados a partir da aplicação da técnica projetiva do Rorschach apresentam um consenso na descrição de profundo comprometimento afetivo, cognitivo e em especial a baixa autoestima, que caracteriza o perfil psicológico de pacientes com TA, porém inconclusas em relação à existência ou não de uma personalidade típica relacionada aos TA, logo há uma necessidade de novos estudos, que permitam esclarecer tal lacuna. 


\section{REFERÊNCIAS}

Alba, C.G. (2005). Imagen corporal en Anorexia Nerviosa. In Resúmenes del XVIII Congresso Internacional de Rorschach y Métodos Proyectivos (p. 322). Barcelona.

American Psychiatric Association (2002). Manual diagnóstico e estatístico de transtornos mentais - DSM-IV-TR (D. Batista, Trans.), $4^{\mathrm{a}}$ ed.

Amorim, P. (2000). Mini (Trabalho original publicado em 2001). Porto Alegre: Editora Artmed.

American Psychiatric Association Practice Guidelines. (2006). Practice Guideline for the Treatment of International Neuropsychiatric Interview (MINI): validation of a short structured diagnostic psychiatric interview. $R e$ vista Brasileira de Psiquiatria, 22(3), 106-115.

Andrade, D., \& Angerami, E. L. S. (2001), The self-esteem of adolescents with and without a cleft lip and/or palate. Revista Latino-Americana de Enfermagem, 9(6), 37-41.

André, C., \& Lelord, F. (2003). Auto-estima: amar a si mesmo para conviver melhor com os outros. Rio de Janeiro: Nova Era.

Appolinario J. C., Cordás T. A., \& Claudino A. M. (2002). Apresentação. Revista Brasileira de Psiquiatria, 24, 1-2.

Avanci, J. Q., Assis, S. G., Santos, N. C., \& Oliveira, R. V. C. (2006). Adaptação transcultural de escala de autoestima para adolescentes. Psicologia Reflexão e Crítica, 20, 397-405.

Anzieu, D. (1989). Os Métodos Projectivos. Rio de Janeiro, Editora Campos.

Bighetti, F. (2003). Tradução e validação do Eating Attitudes Test: EAT-26 em adolescentes do sexo feminino na cidade de Ribeirão Preto-SP. Universidade de São Paulo, Ribeirão Preto.

Bighetti, F., Santos, C. B., Santos, J. E., \& Ribeiro, R. P. P. (2004). Tradução e validação do Eating Attitudes Test em adolescentes do sexo feminino de Ribeirão Preto-SP. J Bras Psiquiatr, 53, 339-46.

Blatt, S. J., \& Lerner, H. (1983). The psychological assessment of object representation. Journal of Personality Assessment, 47(1), 7-28.

Bruch, H. (1982). Psychoterapy in anorexia nervosa. International Journal of Eating Disorders 1(4), 3-14.

Bucaretchi, H. A. (2003). Anorexia e Bulimia Nervosa: a constituição psíquica. In: Anorexia e bulimia nervosa: uma visão multidisciplinar. São Paulo: Casa do Psicólogo.

Camargo, I. (2003). Anorexia e Bulimia: O negativo do corpo - um colar de pérolas sem fio. Em: Anorexia e bulimia nervosa: uma visão multidisciplinar. São Paulo: Casa do Psicólogo.

Claudino, A. M., \& Borges, M. B. F. (2002) Critérios diagnósticos para os transtornos alimentares: conceitos em evolução. Revista. Brasileira de. Psiquiatria, 24: 7-12.

Chabert, C. (2000). A psicopatologia à prova no Rorschach, Lisboa, Climepsi Editores.
Connan, F. (2007). Poor social comparison and the tendency to submissive behavior in anorexia nervosa. International Journal of Eating Disorders, 40(8): 733-739.

Cordás, T. A. (2004). Transtornos alimentares: classificação e diagnóstico. Revista de Psiquiatria Clínica, 31(4), 154-157.

Ferreira, J. E. S., \& Veiga, G. V. (2008). Eating disorder risk behavior in Brazilian adolescents from low socioeconomic level. Appetite, 51, 249-255.

Garcia, A. M., \& Simone, C. J, (2003). Some features of mother of patients with eating disorders. Eating and Weight Disorders, 8(3), 225-230.

Garcia-Alba, C. (2004). Anorexia and depression: depressive comorbidity in anorexic adolescents. The Spanish Journal of Psychology, 7(1): 40-52.

Garner D., \& Garfinkel P. (1979). The Eating Attitudes Test: an index of the symptoms of AN. Psychology Medicine, 9, 273-279.

Ghaderi A., \& Scott B. (2001). Prevalence, incidence and prospective risk factors for eating disorders. Acta Psychiatrica Scandinavica, 104(2), 122-30.

Gorgati, S. B., Holcberg, \& Oliveira (2002). Abordagem psicodinâmica no tratamento dos transtornos alimentares. Revista Brasileira de. Psiquiatria, 24, 44-48.

Heatherton, T. F., \& Wyland, C. (2003). Assessing selfesteem. Em: S. Lopez and R. Snyder. (Eds). Assessing positive psychology (219-233). Washington, DC: APA.

Huke, K., \& Slade P. (2006). An exploratory investigation of the experiences of partners living with people who have bulimia nervosa. European Eating Disorders Review, 14(1): 436-447.

Hutz, C. S. (2000). Adaptação da escala de autoestima de Rosenberg. Manuscrito não publicado. Universidade Federal do Rio Grande do Sul. Porto Alegre, RS.

Hutz, C. S., \& Zanon, C. (2011). Revisão da apadtação, validação e normatização da escala de autoestima de Rosenberg. Aval. Psicol., 10(1): 41-49.

Klein, D. A., \& Walsh, T. (2004). Eating Disorders: Clinical Features and Pathophysiology. Phisiology \& Behaviour 81(2): 359-74.

Jaeger, B., Ruggiero, G. M., Edlund, Gómez-Perreta, C., Lang, F., Mohammadkhani, P., Sahleen-Veasey, H., Schomer, H., \& Lamprecht, F. (2002). Body Dissatisfaction and its interrelations with other risk factors for bulimia nervosa in 12 countries. Psychoterapy and Psychosomatics, 71: 54-61.

Kugu, N., Akyuz, G., Dogan, O., Ersan, E., \& Izgic, F. (2006). The prevalence of eating disorders among university students and the relationship with some individual characteristics. The Australian and New Zealand Journal of Psychiatry, 40(2): 129-135.

McGee, R., \& Williams, S. (2000). Does low self-esteem predict compromising behaviours among adolescents? Journal of Adolescence 23(5), 569-82. 
Melin, P., \& Araújo, A. (2002). Transtornos alimentares em homens: um desafio diagnóstico. Revista Brasileira de Psiquiatria, 24, 73-76.

Mintz, L. B., \& O'Halloran, M. S. (2000). The Eating Attitudes Test: validation with DSM-IV Eating Disorders Criteria. J Pers Assess, 74, 489-503.

Morgan, C. M., Vecchiatti, I. R., \& Negrão, A. Z. (2002). Etiologia dos transtornos alimentares: aspectos biológicos, psicológicos e sócio-culturais. Revista Brasileira de Psiquiatria, 24, 18-23.

Nascimento, R. S. G. F. (2007). Rorschach Comprehensive System data for a sample of 409 adult nonpatients from Brazil. Journal of Personality Assessment, 89 (Suppl. 1), 35-41.

Newn, K., Bell, L., \& Thomas, S. (2003). The impact of a self-esteem group for people with eating disorders: an uncontrolled study. Clinical Psychology and Psychoterapy 10, 64-68.

Nunes, M. A., Bagatini L., Abuchaim A. L., Kunz A., Ramos D., \& Silva, J. (1994). Distúrbios da conduta alimentar: considerações sobre o teste de atitudes alimentares (EAT-26). Revista ABP-APAL, 16, 7-10.

Nunes, M. A., Barros, F. C., Olinto, M. T. A., Camey, S., \& Mari, J. D. J. (2003). Prevalence of abnormal eating behaviors and inappropriate methods for weight control in young women from Brazil: a population - based study. Eat Weight Disord, 8, 100-106.

Nunes, M. A., Camey, S., Olinto, M. T. A., \& Mari, J. J. (2005). The validity and 4-year test-retest reliability of the Brazilian version of the Eating Attitudes Test-26. Braz J Med Biol Res. 38(11), 1.655-1.662.

O'Dea, J. A., \& Abraham, S. (2000). Improving the body image, eating attitudes and behaviors of young male and female adolescents: a new educational approach that focuses on self-esteem. Internacional Journal of eating Disorders, 28, 43-57.

Oliveira, E. A., Santos, M. A. (2006). Perfil psicológico de pacientes com anorexia e bulimia nervosas: a ótica do psicodiganóstico. Medicina, Ribeirão Preto, 39(3): 353360.

Oliveira-Cardoso, E. A., \& Santos, M. A. (2012). Avaliação psicológica de pacientes com anorexia e bulimia nervosas: indicadores do Método de Rorschach. Fractal, Rev. Psicol., 24(1): 159-174.

Organização Mundial de Saúde - OMS (1993). Classificação de transtornos mentais e de comportamento - CID10 (D. Caetano, Trad.). Porto Alegre: Artes Médicas. (Trabalho original publicado em 1992).

Perry, J., Silvera, D., \& Rosenvinge, J. H. (2002). Are oral, obsessive, and hysterical personality traits related to disturbed eating patterns? A general population study of
6,313 men and women. Journal of Personality Assessment 78(3), 405-416.

Ramalho, R. M. (2001). Uma melancolia tipicamente feminina. Revista da APPOA, 20, 37-56.

Rosenberg, M. (1989). Society and the adolescent selfimage. New Jersey: Princeton University Press.

Salmivalli, C., Kaukiainen, A., Kaistaniemi, L., \& Lagerspetz, K. M. (1999) Self-evaluated self-esteem, peerevaluated self-esteem, and defensive egotism as predictors of adolescents' participation in bullying situations. Personality and Social Psychology Bulletin, 25, 1.2681.278 .

Salmon, P., Arnold, J. M., \& Collyer Y. M. (1972). What do the determinants determine: The internal Validity of the Rorschach. Journal of Personality Assesssment, 36(1), 33-38.

Sbicigo, J. B., Bandeira, D. R., \& Dell' aglio, D. D. (2010). Escala de Autoestima de Rosenberg (EAR): validade fatorial e consistência interna. Psico-USF, 15(3): 395-403.

Smith, J. E., Hillard, M. C., \& Rolla, S. (1991). Rorschach evaluation of adolescent bulimics. Adolescence, 26(103): 687-696.

Smith, J. E. (1991). Rorschach assessment of purging and nonpurging bulimics. Journal of Personality Assessment, 56(2): 277-288.

Troise, A. (2006). Body dissatisfaction in women with eating disorders: relationship to early separation anxiety and insecure attachment. Psychosomatic Medicine, 68(1): 449-453.

Vaz, C. E. (1997). O Rorschach: Teoria e Desempenho. Barueri: Manole.

Vaz, C. E. (2006). O Rorschach: Teoria e Desempenho II. São Paulo: Casa do Psicólogo.

Vilella, J. E. M., Lamounier, J. A., Dellaretti, M. A., Neto, J. R. B., \& Horta, G. M. (2004). Transtornos alimentares em escolares. J Pediatr, 80, 49-54.

Wallach, J. D., \& Lowenkopf, E. L. (1986). Five bulimic women: MMPI, Rorschach, and TAT characteristics. International Journal of Eating Disorders, 3(4): 53-66.

Ward, A. (2000). Attachment patterns in eating disorders: past in the present. International Journal of Eating Disorders, 28(4): 370-376.

Weisberg, L. J. Norman, D. K., \& Herzog, D. B. (2006). Personality functioning in normal weight bulimia. International Journal of Eating Disorders, 6(5): 615-631.

Recebido em: 22/07/2009 Revisões Requeridas em: 17/06/2013 Aceito em: 29/03/2014 